\title{
YIELD LOCI CALCULATION OF HEXAGONAL MATERIALS USING A SELFCONSISTENT POLYCRYSTALLINE MODEL
}

\author{
R. A. LEBENSOHN and C. N. TOMÉ* \\ Instituto de Física Rosario (UNR-CONICET) - 27 de Febrero 210 Bis \\ - 2000 Rosario, Argentina \\ *Permanent Address: AECL Research, Whiteshell Laboratories, \\ ROE ILO Manitoba, Canada.
}

(Received 8 November 1995)

\begin{abstract}
Polycrystalline models are used for the calculation of the yield loci of hcp aggregates with different crystallographic textures. Predictions are made using both a classical Taylor Full Constraints (FC) approach and a viscoplastic selfconsistent (VPSC) formulation. Typical deformation modes for low and high temperature are assumed to be active. The vertex configuration of the Single Crystal Yield Surface (SCYS) and the relative activity of different deformation modes as a function of the applied stress are also presented and discussed. For low temperature, when twinning is active, a non centro-symmetric behavior is observed in textured polycrystals along the direction of the $\langle\mathrm{c}\rangle$-axis texture component. Such effect, associated with the directionality of twinning, is well reproduced by the VPSC model. For high temperature, the predicted yield loci are centro-symmetrical but also strongly dependent on the texture of the aggregate.
\end{abstract}

KEY WORDS: Polycrystal yield surface, viscoplastic selfconsistent model, twinning, plastic anisotropy.

\section{INTRODUCTION}

In the present work, we are concerned with the plastic response of textured hcp polycrystals. Uniaxial tests performed at low temperature on these materials demonstrate an appreciable non centro-symmetric response for those orientations of the testing axis along the predominant direction of the crystallographic <c>-axis. Picklesimer (1966) reported the appearance of this strength differential in Zrly-2 tubes with a strong concentration of basal poles at each of the principal directions of the tube. Similar measurements were reported for titanium (Lee and Backofen, 1966) and magnesium (Kelley and Hosford, 1968). This effect is a consequence of the directional dependence of twinning with the sense of the applied stress (Hosford, 1993). Twinning is an important deformation mechanism for low temperature plastic deformation in most hcp crystals. This clear-cut evidence on the relation between micro and macroplasticity could be regarded as a benchmark for testing different micro-macro approaches for modelling mechanical behavior of polycrystals.

The macroscopic plastic behavior of a polycrystal is a consequence of its crystallographic texture and the plastic properties of the constituent grains. Plasticity at the grain level depends on the topology of the Single Crystal Yield Surface (SCYS), a faceted polyhedron in the 5-dim stress space, which defines the locus of possible 
yield states. Tomé and Kocks (1985) analyze the SCYS of hcp in detail, and propose a systematics for grouping the vertices and for characterizing its topology according with the crystal symmetry and the Critical Resolved Shear Stress (CRSS) on the deformation systems. This method - which is specially useful for hcp crystals, due to the large number of potentially active deformation modes with different CRSS's - allows to define topological domains of CRSS's where the vertex configuration remains invariant. For CRSS's within such domains the resultant textures are invariant when the Taylor Full Constraints (FC) approach is used, and do not show large variation when using a selfconsistent scheme. The topological analysis of the SCYS of hcp crystals is performed by Tomé and Kocks (1985) and Tomé et al. (1991), considering different combinations of active deformation modes.

The Polycrystal Yield Surface (PCYS) is the locus of yield states associated with an aggregate of grains, and can be calculated by means of polycrystalline models. Within this type of approach, the effect of texture on polycrystal plasticity can be taken into account. The PCYS is built by probing the polycrystal in different strain directions and using a polycrystalline model to obtain the associated average stress over all the grains of the aggregate. The resulting stress defines a point in the 5-dimensional deviatoric stress space belonging to the PCYS, while the strain vector defines the normal to the PCYS surface at that point. Hence, for each applied strain tensor, the equation of a plane in the stress space is obtained. The inner envelope defined by the resulting set of planes is a good approximation to the actual PCYS. This approach has been used to calculate the PCYS of textured hexagonal (Tomé and Kocks, 1985) and cubic aggregates (Canova and Kocks, 1984; Canova et al., 1985) using a Full Constraints polycrystal model. In particular, the work of Canova and Kocks (1984) on rolled fcc metals shows that the use of a viscoplastic approach in combination with a criterion of equal dissipated energy leads, for materials having a small rate-sensitivity, to essentially the same results as in the case of a rate-insensitive material. Canova (1988) also investigated the effect of grain shape on plastic anisotropy using a RC approach (Honneff and Mecking, 1981). Tomé et al. (1988) use a viscoplastic selfconsistent model based on an isotropic Homogeneous Equivalent Medium (HEM) assumption (Molinari et al., 1987) for the analysis of the plastic properties of Zrly-4 sheets. In this way, they are able to account for the effect of the high intrinsic plastic anisotropy of hcp single crystals on the macroscopic behavior. More recently, Lebensohn and Tomé (1993) use a fully anisotropic viscoplastic selfconsistent (VPSC) formulation to predict the PCYS of rolled Zrly-4 samples at low temperature, finding a good agreement with the yield stresses measured in uniaxial tests performed on samples cut along the principal directions of the sheet. In all the previous cases, the use of rigid-plastic or viscoplastic models amounts to neglect the effect of anisotropic elasticity and internal stresses on yielding. As a consequence, the calculated polycrystal yield surfaces are consistent with a large offset-strain criterion for yielding (i.e.: all grains in the polycrystal are in the fully plastic condition). The same assumption is made in this work.

In what follows, we show predicted PCYS's corresponding to several textures, obtained by means of the FC and VPSC models. Different combinations of slip and twinning systems with different associated CRSS's were selected for the simulations. These combinations were chosen because they give good agreement with experimental evidence obtained for $\mathrm{Zr}$ deformed at low (Tenckhoff, 1978) and high temperature (Akhtar, 1973), respectively. In addition, these sets of active deformation modes have already shown to be adequate for the simulation of deformation textures of $\mathrm{Zr}$ and Zr alloys (Lebensohn and Tomé, 1993; 1994; Lebensohn et al., 1994). 


\section{RESULTS}

Figure 1 shows the basal pole figures corresponding to three possible fabrication textures of $\mathrm{Zr}$-alloy tubes. These textures - labeled A (axial), R (radial) and $\mathrm{T}$ (tangential) were constructed so as to have most of the crystallographic $<c>$-axis aligned with the principal directions of the tube. These textures were generated assigning appropriate weights to 1944 fixed orientations in Euler space. The three textures, and also a random distribution of orientations, are used as input for the PCYS calculations that follow.

Let us analyze the low temperature case. Experiments done by Tenckhoff (1978) have shown that $\{10 \overline{1} 0\}<1 \overline{2} 10\rangle$ prismatic slip $(\mathrm{pr}<\mathrm{a}>)$ together with $\{10 \overline{1} 2\}<\overline{1} 011\rangle$ tensile twinning (ttw) and $\{2 \overline{1} \overline{1} 2\}<2 \overline{1} \overline{1} 3>$ compressive twinning (ctw) are active in coarse grained $\mathrm{Zr}$ polycrystals at low temperature. The complete set of systems associated with these deformation modes is listed in Table 1, together with the notation convention used (after Tomé and Kocks, 1985). Although the actual values of the CRSS's associated with these modes depend on different metallurgical variables - grain-size, alloy-content,

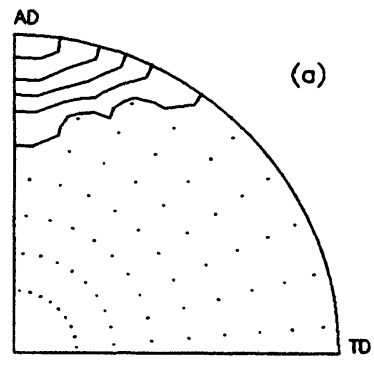

TEX A

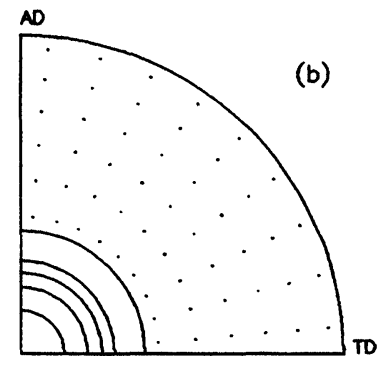

TEX R

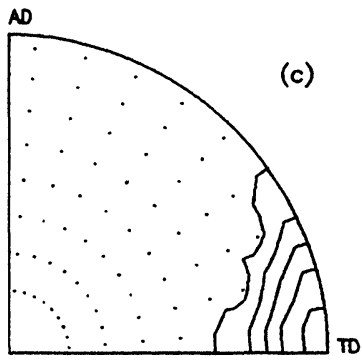

TEX T

Figure 1 Basal pole figures for heavily textured tubes. Crystallographic $\langle\mathrm{c}>$-axis are predominantly aligned with: a) axial direction (A), b) radial direction ( $R$ ), c) tangential direction (T).

Table 1 Prismatic slip and twinning systems in hcp materials.

\begin{tabular}{|c|c|c|c|c|c|}
\hline \multicolumn{2}{|c|}{$t t w$} & \multicolumn{2}{|c|}{$c t w$} & \multicolumn{2}{|c|}{$p r<a>$} \\
\hline system & $n-b$ & system & $n-b$ & system & $n-b$ \\
\hline$+\mathrm{ZO}$ & $(10 \overline{1} 2)[\overline{1} 011]$ & $-Y 1$ & $(2 \overline{1} \overline{1} 2)[2 \overline{1} \overline{1} \overline{3}]$ & $+\mathrm{EB}$ & $(10 \overline{1} 0)[1 \overline{2} 10]$ \\
\hline$+\mathrm{ZP}$ & $(01 \overline{1} 2)[0 \overline{1} 11]$ & $-\mathrm{Y} 2$ & $(11 \overline{2} 2)[11 \overline{2} \overline{3}]$ & $-E B$ & $(10 \overline{1} 0)[\overline{1} 2 \overline{1} 0]$ \\
\hline$+Z Q$ & $(\overline{1} 102)[1 \overline{1} 01]$ & $-Y 3$ & $(\overline{1} 2 \overline{1} 2)[\overline{1} 2 \overline{1} \overline{3}]$ & $+\mathrm{FB}$ & $(01 \overline{1} 0)[2 \overline{1} \overline{1} 0]$ \\
\hline$+Z R$ & $(\overline{1} 012)[10 \overline{1} 1]$ & $-\mathrm{Y} 4$ & $(\overline{2} 112)[\overline{2} 11 \overline{3}]$ & $-\mathrm{FB}$ & $(01 \overline{1} 0)[\overline{2} 110]$ \\
\hline$+Z S$ & $(0 \overline{1} 12)[01 \overline{1} 1]$ & $-\mathrm{Y} 5$ & $(\overline{1} \overline{1} 22)[\overline{11} 2 \overline{3}]$ & $+\mathrm{GB}$ & $(\overline{1} 100)[11 \overline{2} 0]$ \\
\hline$+\mathrm{ZT}$ & $(1 \overline{1} 02)[\overline{1} 101]$ & $-Y 6$ & $(1 \overline{2} 12)[1 \overline{1} 1 \overline{3}]$ & $-G B$ & $(\overline{1} 100)[\overline{1} \overline{1} 20]$ \\
\hline
\end{tabular}


applied strain-rate, microstructure, etc. - the experimental evidence (Tenckhoff, 1974) indicates that the following relation holds: $\tau^{\mathrm{pr}<\mathrm{a}}<\tau^{\mathrm{tw}}<\tau^{\mathrm{ctw}}$. In what follows we adopt the set of CRSS's used by Lebensohn and Tomé $(1993 ; 1994)$ to explain the formation of typical low temperature $\mathrm{Zr}$ textures: $\tau^{\mathrm{pr}<\mathrm{a}}=1.0, \tau^{\mathrm{tw}}=1.25, \tau^{\mathrm{ctw}}=2.5$ (arbitrary units). These ratios of CRSS's (and other values used in this work) have to be regarded as being only qualitative correct. Actual values of CRSS's are strongly dependent on metallurgical and alloying conditions of the polycrystal. The difference between the critical stresses associated with tensile and compressive twinning makes for a high directionality of the single crystal's plastic properties. Since prismatic slip cannot accommodate deformation along the $<\mathrm{c}>$-axis, tensile (compressive) twinning is the only available mechanism providing a positive (negative) strain along the $<\mathrm{c}>$-axis and, as a consequence, the yield stress for a crystal along the $\langle c\rangle$-axis is smaller for a tensile than for a compressive stress state.

The high directionality of the single crystal properties is, in turn, reflected in the macroscopic behavior of heavily textured polycrystals. Figure 2 shows the projections in the axial-tangential plane of the PCYS's calculated with FC and VPSC models for $\mathrm{A}, \mathrm{R}, \mathrm{T}$ and random textures. All curves are normalized to the value of the yield stress for uniaxial tension along the axial direction. The expected strength differential appears clearly for the VPSC curves: the positive values of yield stresses corresponding to uniaxial tests along the axial, radial and tangential directions are lower than the negative ones, for textures A, R, and T, respectively. In addition, the projection of the random PCYS is - as expected - a centro-symmetric curve. It seems evident, on the other hand, that the FC model does not predict any substantial strength differential. The reason can be found analyzing the topology of the SCYS for the selected combination of CRSS's.

Table 2 shows the set of irreducible vertices of the SCYS for $\tau^{\mathrm{pr}<a \mathrm{z}}=1.0, \tau^{\mathrm{tw}}=1.25$, $\tau^{\mathrm{ctw}}=2.5$. The symbol " 1 " indicates that the hyperplane associated with the

Table 2 Set of irreducible vertices associated with the SCYS of an hcp crystal for $\tau^{\text {preas }}=1.0$, $\tau^{t t w}=1.25, \tau^{c t w}=2.5$.

\begin{tabular}{cccccccccccccccc}
\hline$\#$ & & & \multicolumn{1}{c}{$t w$} & & & \multicolumn{1}{c}{$c w w$} & & \multicolumn{3}{c}{$p r<a>$} \\
& $+Z O$ & $+Z P$ & $+Z Q$ & $+Z R$ & $+Z S$ & $+Z T$ & $-Y 1$ & $-Y 2$ & $-Y 3$ & $-Y 4$ & $-Y 5$ & $-Y 6$ & $+E B$ & $+F B$ & $+G B$ \\
\hline 1 & 1 & 1 & 1 & 1 & 1 & 1 & 0 & 0 & 0 & 0 & 0 & 0 & 0 & 0 & 0 \\
2 & 0 & 1 & 1 & 0 & 1 & 1 & 0 & 0 & 0 & 0 & 0 & 0 & 0 & 1 & 1 \\
3 & 0 & 0 & 0 & 1 & 1 & 1 & 0 & 0 & 0 & 0 & 1 & 1 & 0 & 0 & 0 \\
4 & 0 & 0 & 1 & 1 & 1 & 1 & 0 & 0 & 0 & 0 & 1 & 0 & 0 & 0 & 0 \\
5 & 0 & 0 & 1 & 0 & 1 & 1 & 0 & 0 & 0 & 0 & 1 & 0 & 0 & 1 & 0 \\
6 & 0 & 0 & 0 & 1 & 1 & 0 & 0 & 0 & 0 & 0 & 1 & 1 & 0 & $\frac{1}{1}$ & 0 \\
7 & 0 & 0 & 0 & 1 & 1 & 0 & 0 & 0 & 0 & 0 & 1 & 0 & 1 & 1 & 0 \\
8 & 0 & 0 & 0 & 1 & 0 & 1 & 0 & 0 & 0 & 0 & 1 & 1 & $\frac{1}{1}$ & 0 & 1 \\
9 & 0 & 0 & 1 & 0 & 0 & 1 & 0 & 0 & 0 & 0 & 1 & 0 & 1 & $\frac{1}{1}$ & 0 \\
10 & 0 & 0 & 0 & 0 & 1 & 0 & 0 & 0 & 0 & 0 & 1 & 1 & 1 & $\frac{1}{1}$ & 1 \\
11 & 0 & 0 & 0 & 1 & 0 & 0 & 0 & 0 & 0 & 0 & 1 & 1 & 0 & 1 \\
12 & 0 & 0 & 0 & 0 & 1 & 0 & 0 & 0 & 0 & 0 & 1 & 1 & 1 & 0 & 1 \\
& & & & & & & & & & & & & & & \\
13 & 0 & 0 & 0 & 0 & 0 & 0 & 1 & 1 & 1 & 1 & 1 & 1 & 0 & 0 & 0 \\
14 & 0 & 0 & 0 & 0 & 0 & 0 & 1 & 0 & 0 & 1 & 1 & 1 & 1 & 0 & 1 \\
15 & 0 & 0 & 0 & 0 & 0 & 0 & 0 & 0 & 0 & 1 & 1 & 1 & 1 & $\frac{1}{1}$ & 0 \\
16 & 0 & 0 & 0 & 0 & 0 & 0 & 1 & 0 & 1 & 1 & 0 & 1 & 1 & 1 & 0 \\
\hline
\end{tabular}




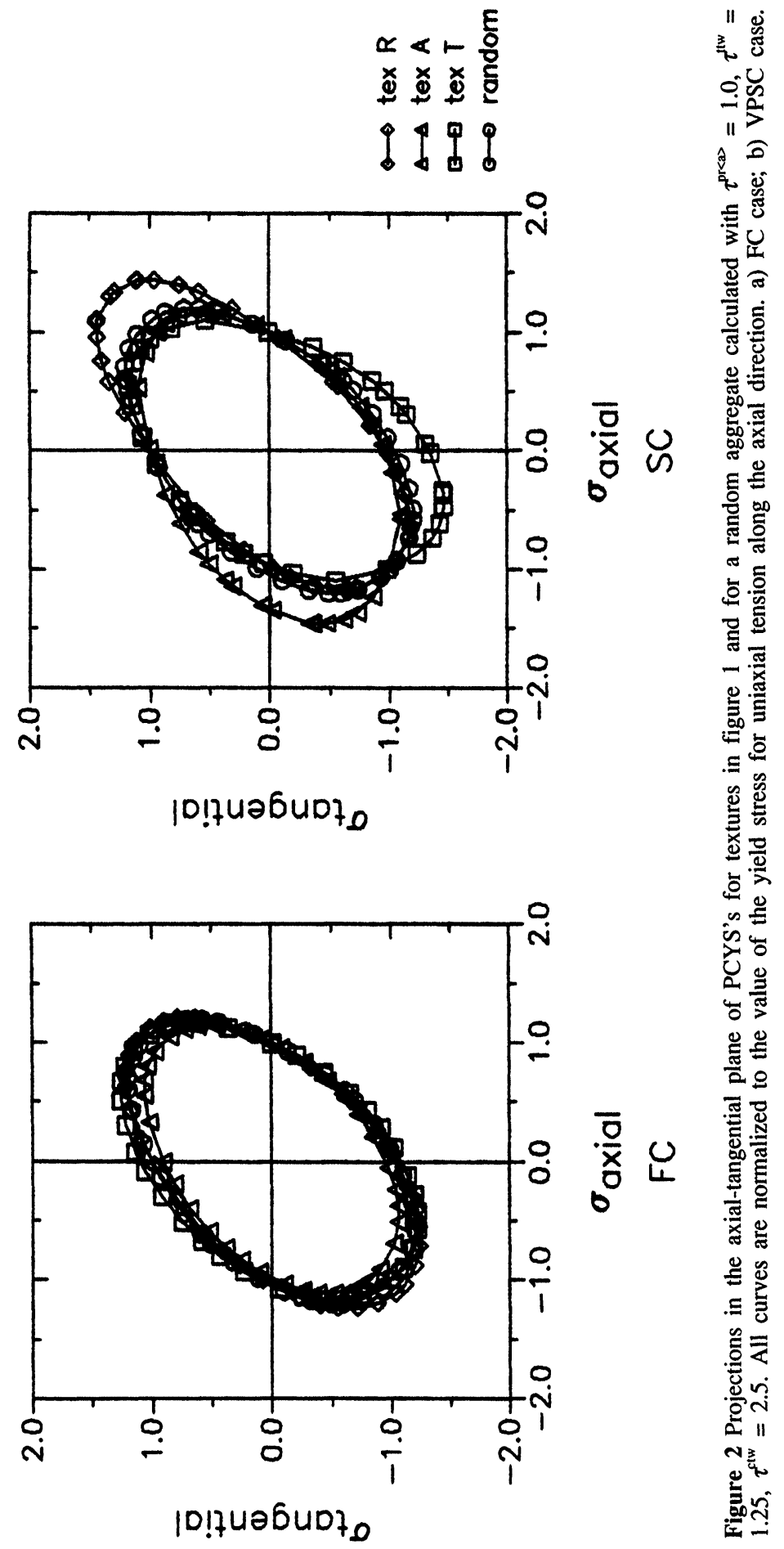


corresponding system participates in the formation of the vertex while the symbol " $\overline{1}$ " indicates that the opposite system participates (i.e.: $-E B$ is opposite to +EB). The characteristic of the irreducible vertices is that they contain all the information concerning the SCYS, and the remaining vertices of the SCYS can be generated by applying the symmetry operations of the hexagonal crystal to the irreducible ones (Tomé and Kocks, 1985). For the particular configuration considered here the SCYS is formed by 104 vertices, out of which only 16 are irreducible. It can be seen that most vertices in the set are formed by facets associated with both tensile and compressive twinning systems. Only vertices \#1 to \#2 and \#13 to \#16 include either ttw or ctw systems, respectively. Within the FC model, imposing the five deviatoric strain components to each grain requires that the yield stress coincides with the vertex of the SCYS that contains the applied strain inside its "cone of normals". As a consequence, if within the FC calculation the applied strain determines - for a given grain - the activation of a vertex of type \#3 to \#12, tensile and compressive twins will be generated simultaneously in such grain. This result is contradictory with the experimental evidence (Tenckhoff, 1978). The VPSC model, on the other hand, allows strain incompatibilities to be accommodated by the grain's surroundings, according to the strength of the interaction between grain and matrix. Hence, the local stress state does not need to be located in a vertex of the SCYS, and is usually located at a facet of the yield surface. In this way, the grains deform without "mixing" the two types of twinning systems, and stress discontinuities between grains are less severe than in the FC approach.

The topological analysis of the SCYS may be complemented by the study of the stress state associated with each vertex. The stresses are expressed in the crystal reference frame defined by $x_{1} \equiv[1 \overline{2} 10], x_{2} \equiv[10 \overline{1} 0]$ and $x_{3} \equiv[0001]$ and the stress tensor is expanded in the basic of symmetric orthonormal deviatoric tensors $\mathbf{b}^{(\lambda)}$ :

$$
\begin{array}{ll}
\mathbf{b}^{(1)}=\frac{1}{\sqrt{2}}\left[\begin{array}{ccc}
-1 & 0 & 0 \\
0 & 1 & 0 \\
0 & 0 & 0
\end{array}\right] & \mathbf{b}^{(2)}=\frac{1}{\sqrt{6}}\left[\begin{array}{ccc}
-1 & 0 & 0 \\
0 & -1 & 0 \\
0 & 0 & 2
\end{array}\right] \\
\mathbf{b}^{(3)} & =\frac{1}{\sqrt{2}}\left[\begin{array}{lll}
0 & 0 & 0 \\
0 & 0 & 1 \\
0 & 1 & 0
\end{array}\right] \\
\mathbf{b}^{(5)} & =\frac{1}{\sqrt{2}}\left[\begin{array}{lll}
0 & 1 & 0 \\
1 & 0 & 0 \\
0 & 0 & 0
\end{array}\right]
\end{array}
$$

The tensors $\mathbf{b}^{(\lambda)}$ represent five independent shears : $\mathbf{b}^{(2)}$ represents the tetrahedral shear, $\mathbf{b}^{(1)}$ and $\mathbf{b}^{(5)}$ represent prismatic shears, and $\mathbf{b}^{(3)}$ and $\mathbf{b}^{(4)}$ the basal shears. The deviatoric stress tensor may be written as a linear combination of the basis tensors:

$$
\sigma_{\mathrm{ij}}=\sum_{\lambda=1}^{5} \sigma^{(\lambda)} \mathrm{b}_{\mathrm{ij}}^{(\lambda)}
$$

where the components $\sigma^{(\lambda)}$ are a linear function of the $\sigma_{\mathrm{ij}}$, and define a 5-dimensional vector of the form: 


$$
\sigma=\left[\frac{1}{\sqrt{2}}\left(\sigma_{22}^{\prime}-\sigma_{11}^{\prime}\right), \frac{\sqrt{3}}{\sqrt{2}} \sigma_{33}^{\prime}, \sqrt{2} \sigma_{23}^{\prime}, \sqrt{2} \sigma_{31}^{\prime}, \sqrt{2} \sigma_{12}^{\prime}\right]
$$

The equivalent stress is defined as:

$$
\sigma_{\mathrm{eq}}=\frac{\sqrt{3}}{\sqrt{2}}\left(\sigma_{\mathrm{ij}}^{\prime} \sigma_{\mathrm{ij}}^{\prime}\right)^{1 / 2}=\frac{\sqrt{3}}{\sqrt{2}}\left(\sigma^{(\lambda)} \sigma^{(\lambda)}\right)^{1 / 2}
$$

Table 3 shows the 5-dim deviatoric stress vectors associated with the irreducible vertices of the SCYS discussed above, together with the corresponding equivalent stress. It can be seen that the vertex \#1 (\#13), formed by the six tensile (compressive) twinning systems, has associated an uniaxial tensile (compressive) stress state along the $c-<a x i s>$. The magnitude of compressive yield stress is higher than the tensile one. The equivalent stresses can be grouped in 3 different intervals, according to their magnitude, as follows: a) "only" ttw (\#1 and \#2), b) "only" ctw (\#13 to \#16) and "mixed" ttw and ctw (\#3 to \#12), making evident another disadvantage of the FC model, i.e: it leads - at least for this set of CRSS's - to severe local stress discontinuities.

Other revealing comparison between FC and VPSC results is shown in figure 3. This figure displays the relative activity of the deformation modes as a function of the angle $\alpha$, formed by the direction of resulting deviatoric stress and a fixed direction in the $\pi$-plane for the axial texture case (texture A, in figure 1$)$. The vertical lines in each graph mark the angle $\alpha$ associated with an uniaxial tensile ( + ) or compressive (-) stress state along the sample's principal directions. It is evident that the statistics are completely different for each type of calculation. In the FC case, the activities of both twinning modes are higher than the prismatic slip activity, irrespective of the orientation of the imposed strain. In the VPSC case, on the other hand, the easiest $\mathrm{pr}<\mathrm{a}>\mathrm{slip}$ is the most

Table 3 Deviatoric stress components in the vector convention of equation (3), and equivalent stresses associated with the set of irreducible vertices listed in Table 2.

\begin{tabular}{lcccccc}
\hline$\#$ & $\sigma^{1}$ & $\sigma^{2}$ & $\sigma^{3}$ & $\sigma^{4}$ & $\sigma^{5}$ & $\sigma_{\text {eq }}$ \\
\hline 1 & 0.00 & 2.05 & 0.00 & 0.00 & 0.00 & 2.51 \\
2 & -0.82 & 1.58 & 0.00 & 0.00 & 1.41 & 2.78 \\
3 & 0.67 & 1.28 & 11.68 & 0.00 & 0.00 & 14.42 \\
4 & 0.49 & 1.49 & 8.51 & 4.92 & 0.84 & 12.24 \\
5 & 0.09 & 1.31 & 7.97 & 4.60 & 1.41 & 11.53 \\
6 & -0.43 & 0.70 & 11.14 & 2.55 & -1.41 & 14.14 \\
7 & -0.82 & 0.63 & 9.53 & 5.50 & -1.41 & 13.65 \\
8 & 1.63 & 1.05 & 10.63 & 0.00 & 0.00 & 13.24 \\
9 & 0.82 & 1.11 & 7.32 & 4.23 & 1.41 & 10.64 \\
10 & -0.82 & 0.49 & 10.95 & 2.55 & -1.41 & 13.92 \\
11 & 0.82 & 0.40 & 9.78 & 2.55 & -1.41 & 12.55 \\
12 & -1.63 & 0.06 & 10.54 & 0.00 & 0.00 & 13.07 \\
& & & & & & \\
13 & 0.00 & -4.53 & 0.00 & 0.00 & 0.00 & 5.55 \\
14 & -1.63 & -3.59 & 2.94 & 0.00 & 0.00 & 6.02 \\
15 & -0.82 & -2.64 & 4.41 & 2.55 & -1.41 & 7.31 \\
16 & -0.82 & -4.06 & 0.00 & 0.00 & -1.41 & 5.36 \\
\hline
\end{tabular}



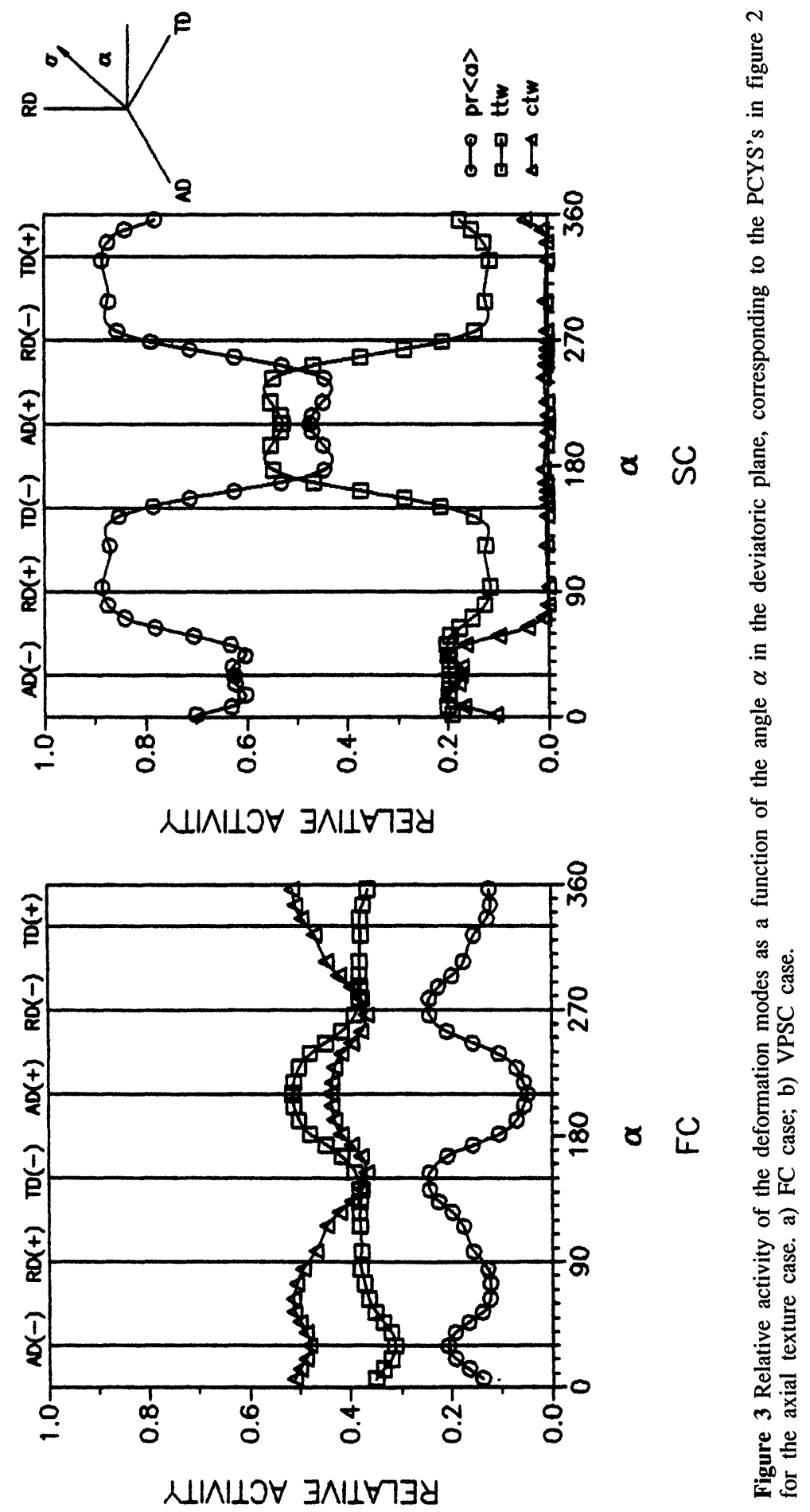
active mode while twinning plays a secondary role. It is specially interesting to regard what happens for angles labeled $\mathrm{AD}(+)$ and $\mathrm{AD}(-)$, corresponding to uniaxial tension and compression along the axial direction, i.e.: the predominant direction of the crystallographic <c>-axis. While in the FC plot, there is only a minor decrease of ctw (ttw) activity for the tensile (compressive) case, the VPSC curves behave as expected, i.e.: the only non-negligible activity of ctw systems is obtained when the stress direction is very close to the $\mathrm{AD}(-)$ while the highest ttw activity is found in the vicinity of the $\mathrm{AD}(+)$ orientation.

The combination of prismatic slip and tensile and compressive twinning is not the only set of active systems consistent with low temperature deformation of $\mathrm{Zr}$ alloys. Depending on the degree of alloying and on metallographic parameters such as the grain size and the dislocation density, there is evidence that $\{10 \overline{1} 1\}<2 \overline{1} \overline{1} \overline{3}\rangle$ pyramidal $<c+a>$ slip (pyr $<c+a>$ ) is active (Pochettino et al., 1992). Moreover, the formation of some typical $\mathrm{Zr}$ alloys textures at low temperature can be explained in terms of a substitution of the ctw mode by the pyr $<c+a>$ mode (Lebensohn and Tomé, 1994). Table 4 shows the notation for the 24 pyr $<c+a>$ systems and table 5 lists the configuration of 37 irreducible vertices of the SCYS (out of a complete set of 317 vertices) associated with the set of CRSS's: $\tau^{\text {pr<a }}=1.0, \tau^{\text {tw }}=1.5, \tau^{\text {yrrecta> }}=4.0$. The SCYS associated with this set of deformation modes has been analyzed in detail by Tomé and Kocks (1985), and the topological domain for the CRSS's considered here is the one that those authors label ' $a_{1}$ '. We can distinguish three types of vertices: a) only ttw (\#1), b) both ttw and pyr<c+a> (\#2 to \#16) and c) no ttw (\#17 to \#37). Unlike the former case, the intersection of ttw and pyr $<c+a>$ facets to form a vertex (v.g.: type (b), \#2 to \#16) is not a priori forbidden for physical reasons.

Also in relation with this example, figure 4 displays the projections of the predicted PCYS for A, R, T and random textures for the aforementioned combination of CRSS's. As in the previous case, this set of CRSS's determines a highly anisotropic plastic behavior due to the difference between the yield stresses of the active mechanisms in tension (i.e.: tensile twinning) and in compression (i.e.: pyr $<c+a>$ slip) along the $<c>-$

Table 4 Pyramidal $<c+a>$ slip systems in hcp materials.

\begin{tabular}{|c|c|c|c|c|c|}
\hline \multicolumn{6}{|c|}{$p y r<c+a>$} \\
\hline$+\mathrm{TO}$ & $(1 \overline{1} 01)[2 \overline{1} \overline{1} \overline{3}]$ & $+P Q$ & $(01 \overline{1} 1)[\overline{1} 2 \overline{1} \overline{3}]$ & $+R B$ & $(\overline{1} 011)[\overline{11} 2 \overline{3}]$ \\
\hline -TO & $(1 \overline{1} 01)[\overline{2} 113]$ & $-P Q$ & $(01 \overline{1} 1)[1 \overline{2} 13]$ & $-R S$ & $(\overline{1} 011)[11 \overline{2} 3]$ \\
\hline$+\mathrm{OT}$ & $(10 \overline{1} 1)[2 \overline{1} \overline{1} \overline{3}]$ & $+Q P$ & $(\overline{1} 101)[\overline{1} 2 \overline{1} \overline{3}]$ & $+\mathrm{SR}$ & $(0 \overline{1} 11)[\overline{11} 2 \overline{3}]$ \\
\hline -OT & $(10 \overline{1} 1)[\overline{2} 113]$ & $-Q P$ & $(\overline{1} 101)[1 \overline{2} 13]$ & $-S R$ & $(0 \overline{1} 11)[11 \overline{2} 3]$ \\
\hline$+\mathrm{OP}$ & $(10 \overline{1} 1)[11 \overline{2} \overline{3}]$ & $+Q R$ & $(\overline{1} 101)[\overline{2} 11 \overline{3}]$ & $+\mathrm{ST}$ & $(0 \overline{1} 11)[1 \overline{2} 1 \overline{3}]$ \\
\hline$-O P$ & $(10 \overline{1} 1)[\overline{1} \overline{1} 23]$ & $-Q R$ & $(\overline{1} 101)[2 \overline{1} \overline{1} 3]$ & $-\mathrm{ST}$ & $(0 \overline{1} 11)[\overline{1} 2 \overline{1} 3]$ \\
\hline$+\mathrm{PO}$ & $(01 \overline{1} 1)[11 \overline{2} \overline{3}]$ & $+R Q$ & $(\overline{1} 011)[\overline{2} 11 \overline{3}]$ & $+\mathrm{TS}$ & $(1 \overline{1} 01)[1 \overline{2} 1 \overline{3}]$ \\
\hline$-\mathrm{PO}$ & $(01 \overline{1} 1)[\overline{1} \overline{1} 23]$ & $-R Q$ & $(\overline{1} 011)[2 \overline{1} \overline{1} 3]$ & $-\mathrm{TS}$ & $(1 \overline{1} 01)[\overline{1} 2 \overline{1} 3]$ \\
\hline
\end{tabular}




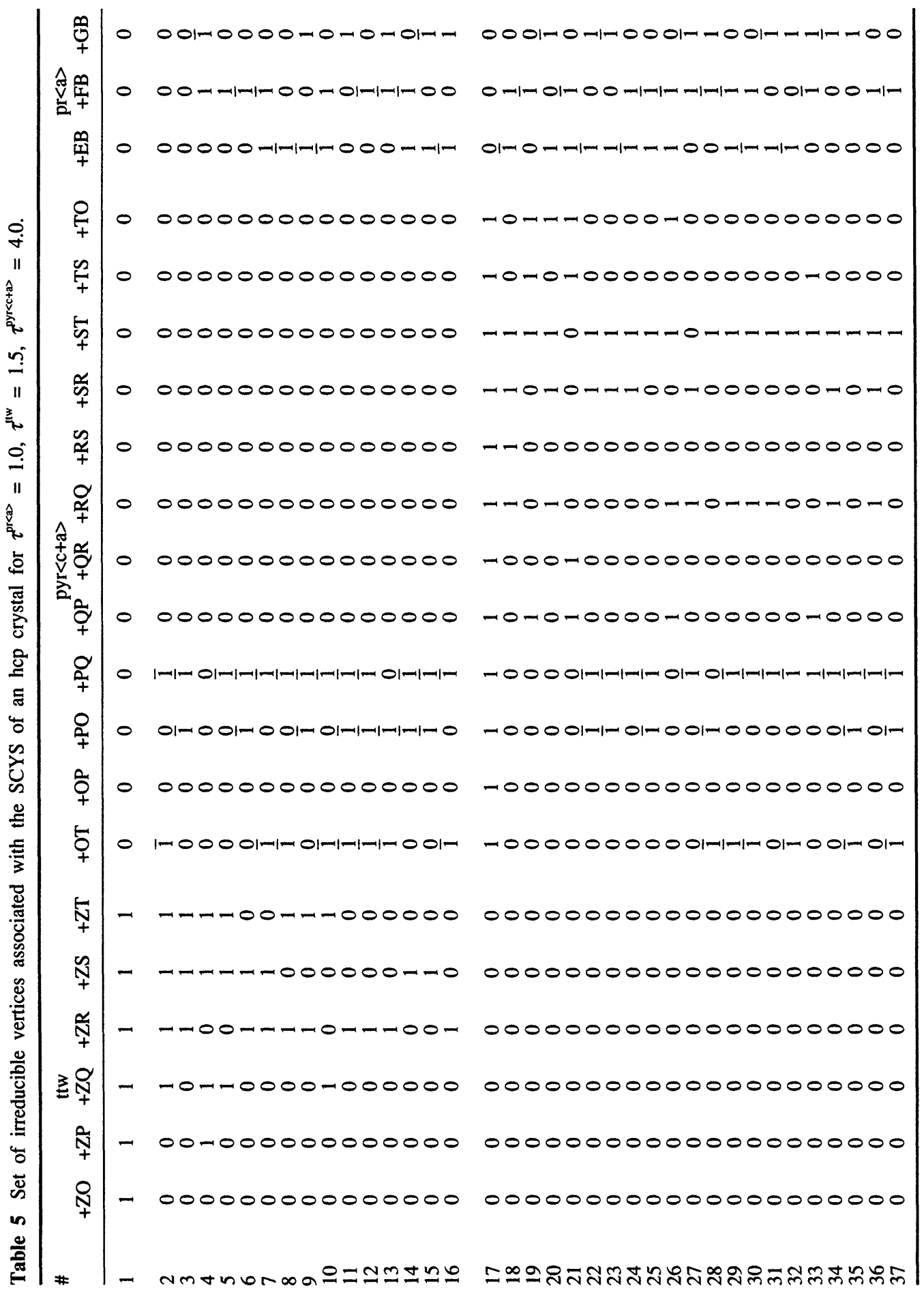



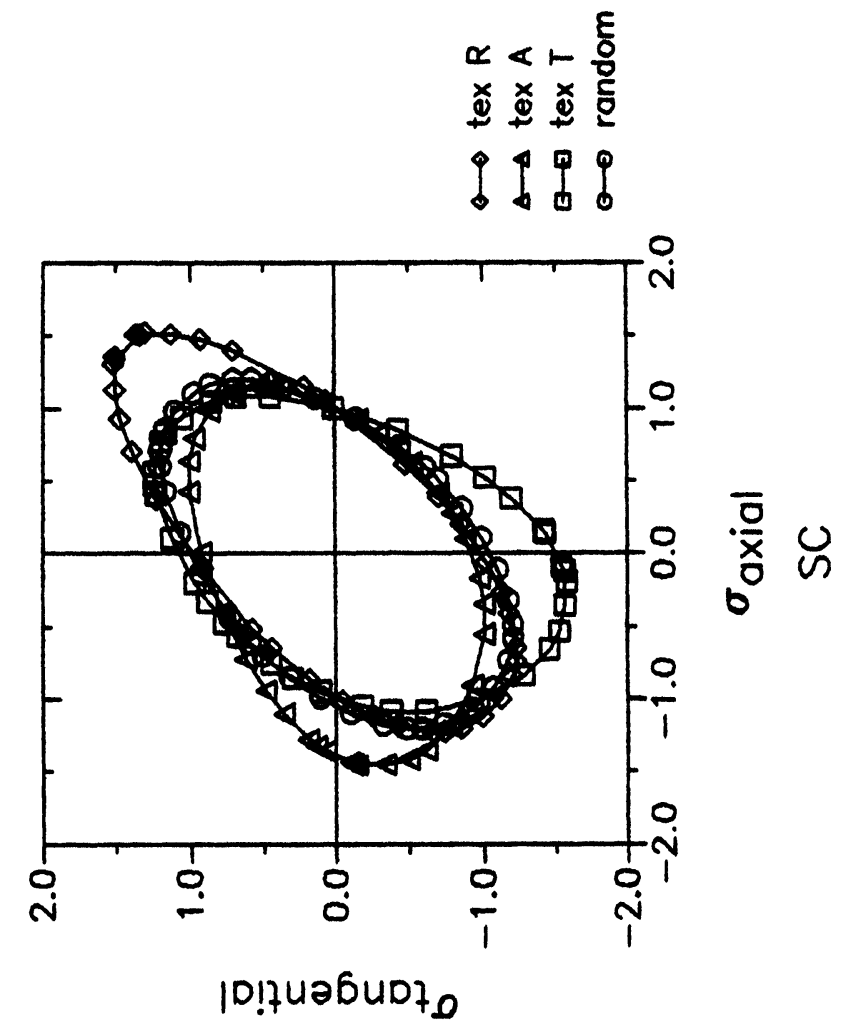

110

○ิ

11 仓ें

色记

กิร

롫

高

政。

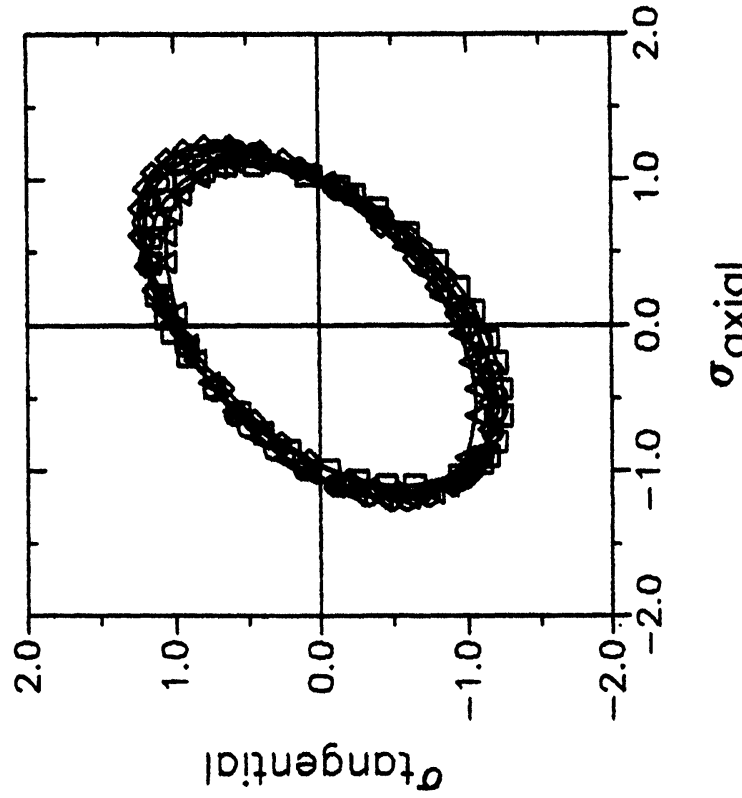

용 
axes. Once again, a marked texture-induced strength differential appears in the VPSC case but not in the FC case. Finally, figure 5 shows the comparison between the relative activities as a function of the applied stress predicted by the FC and the VPSC models for the axial texture case. While the FC model requires a high activity (always $>60 \%$ ) of the hard pyr $<\mathrm{c}+\mathrm{a}>$ mode, within the VPSC formulation most of the deformation can be accommodated by the soft $\mathrm{pr}<\mathrm{a}\rangle$, and the pyr $<\mathrm{c}+\mathrm{a}\rangle$ activity is negligible for the whole range of angles $a$. On the other hand, in the VPSC case, tensile twinning has a minor - but non negligible - contribution to the overall deformation, except for stress states close to uniaxial tension along the axial axis (v.g.: the main orientation of $\langle\mathrm{c}\rangle$-axis) for which an increase of twinning activity is predicted. Evidently, this orientation dependence of tensile twinning activity is responsible for the appearance of the strength differential along the axial direction.

Finally, let us consider the high temperature case. It is known that twinning is not present during the high temperature deformation of hcp materials. Moreover, in the case of high temperature deformation of $\mathrm{Zr}$, the $(0001)<1 \overline{2} 10>$ basal slip $(\mathrm{b}<\mathrm{a}>$ ) together with prismatic $<a>$ and pyramidal $<c+a>$ slip are the available plastic deformation mechanisms (Akhtar, 1973). The six basal systems are listed in table 6. A combination of CRSS's consistent with experimental high temperature textures is: $\tau^{\mathrm{pr}<\mathrm{a}}=1.0, \tau^{\mathrm{b}<\mathrm{a}}$ $=1.5, \tau^{\text {pyr }<\mathrm{c}+2>}=4.0$. (Lebensohn, et al., 1994). The SCYS for this combination contains a total of 266 vertices and the set of irreducible vertices, listed in table 7, contains 17. Four kinds of vertices can be distinguished: a) only pyr $<c+a>(\# 1)$, b) no b $<a>$ (\#2 to \#4), c) no pr<a> (\#5 and \#6) and d) all modes present (\#7 to \#17). The FC and VPSC predictions of the PCYS's associated with the same 4 typical textures used in the former cases are shown in figure 6. In this case, when twinning is not present, the difference between FC and VPSC predictions are not as marked as in the low temperature cases. The PCYS projection is (appreciably) different for each of the four different input textures but, on the other hand, all of them are centro-symmetric, i.e.: the strength differential disappears when twinning is prevented. The centro-symmetry of the predicted PCYS is also reflected in the plots of relative-activity vs. applied stress for the axial texture case (figure 7). Evidently, all curves are periodic with a period of 180 degrees. As in the previous cases, the main difference between the FC and VPSC is in the activity of the hard pyr $<\mathrm{c}+\mathrm{a}>$ mode. While in the FC case it is appreciably high, in the VPSC case it is always negligible except for stress states close to uniaxial tension or uniaxial compression along the basal texture component, when the strain must be mostly accommodated along the <c>-axis of the crystallites.

Table 6 Basal slip systems in hcp materials.

\begin{tabular}{lc}
\hline system & $b<a>$ \\
\hline$+\mathrm{BE}$ & $n-b$ \\
$-\mathrm{BE}$ & $(0001)[1 \overline{2} 10]$ \\
$+\mathrm{BF}$ & $(0001)[\overline{1} 2 \overline{1} 0]$ \\
$-\mathrm{BF}$ & $(0001)[2 \overline{1} \overline{1} 0]$ \\
$+\mathrm{BG}$ & $(0001)[\overline{2} 110]$ \\
$-\mathrm{BG}$ & $(0001)[11 \overline{2} 0]$ \\
\hline
\end{tabular}




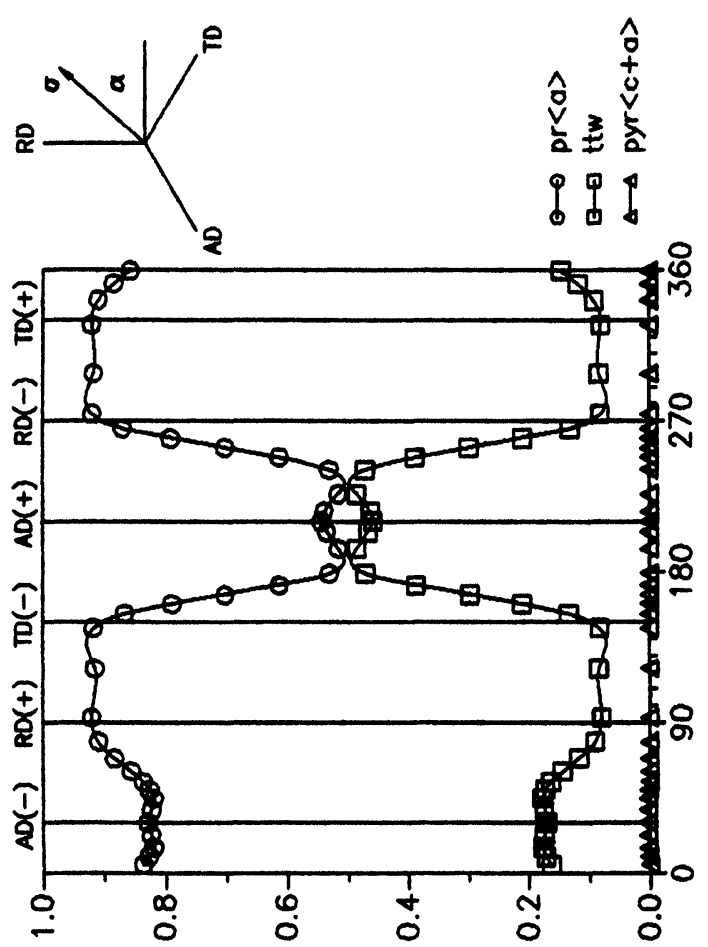

政

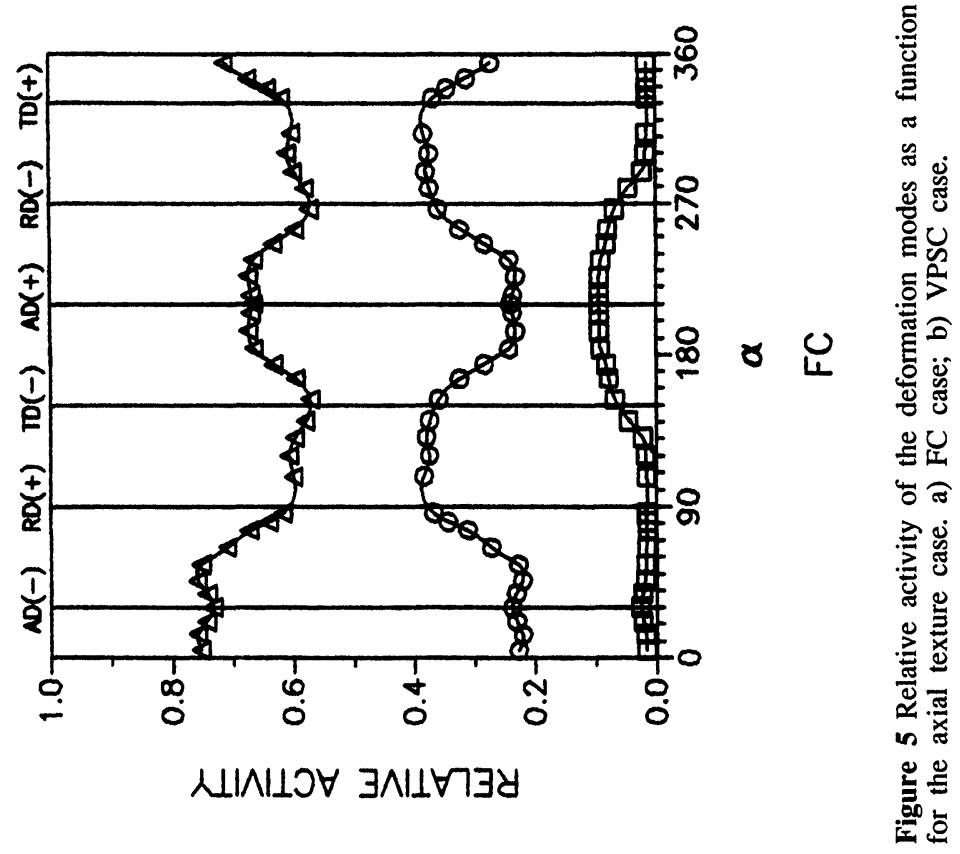




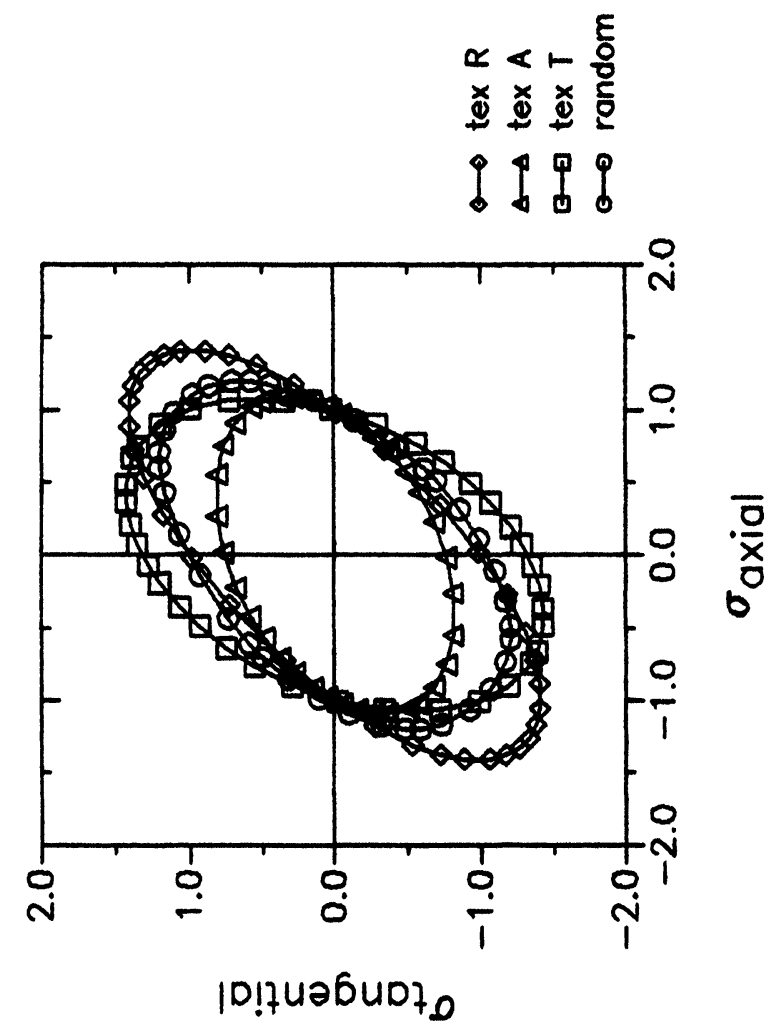

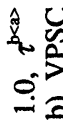

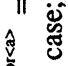

尔

氜施

롫.

폻

$\overline{0} \cup \quad$

o u

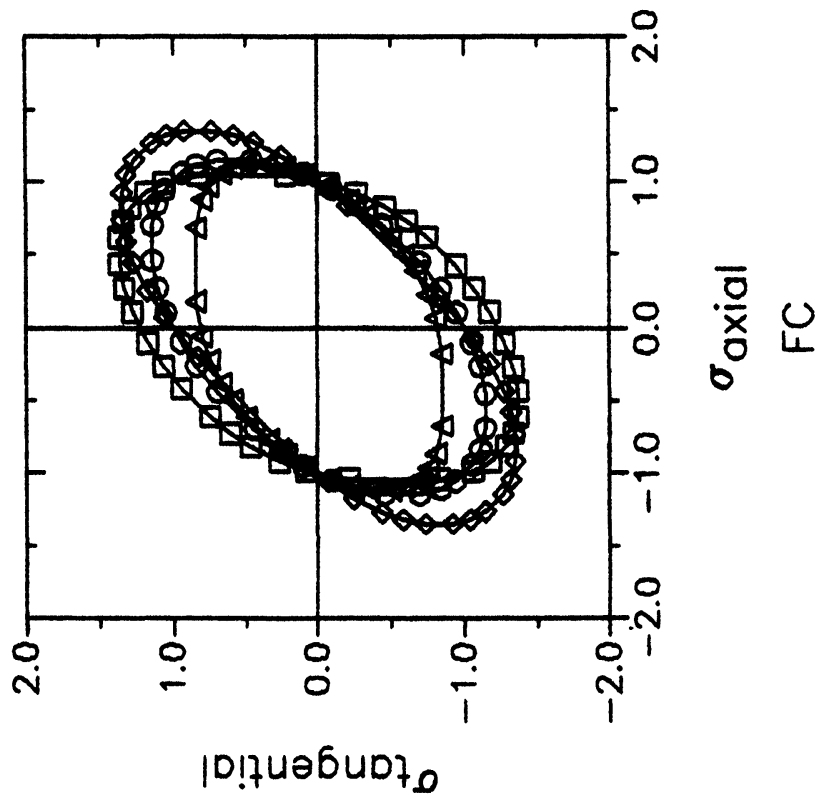

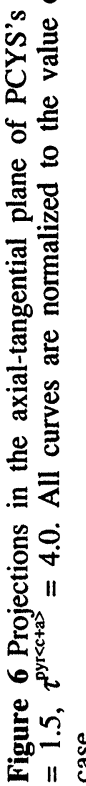



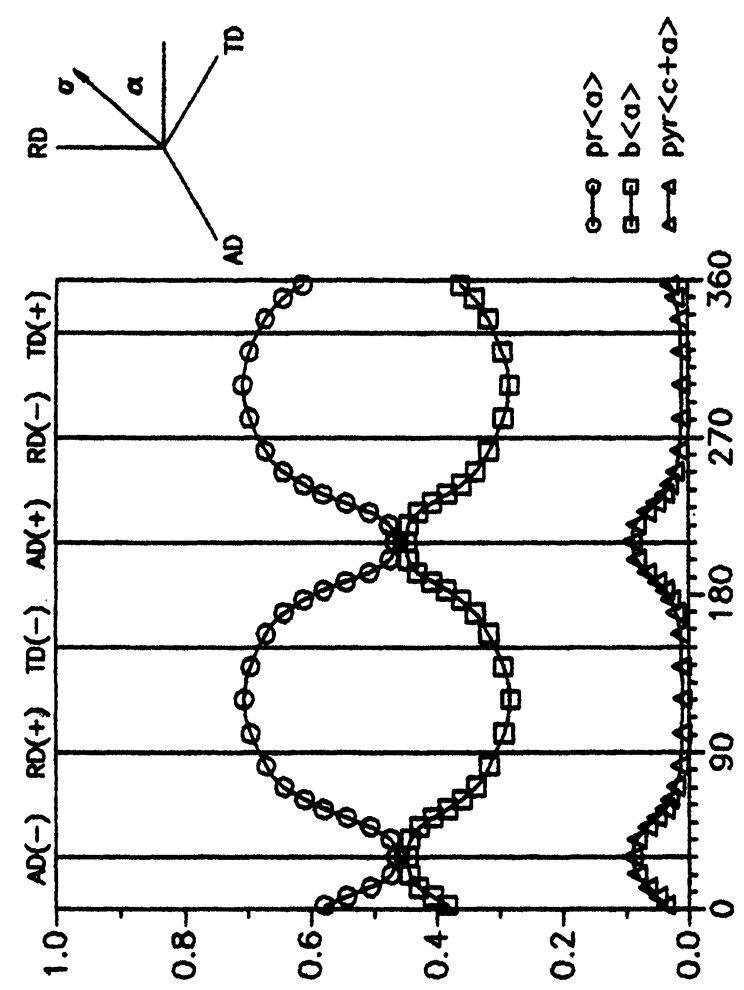

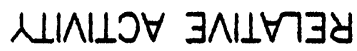

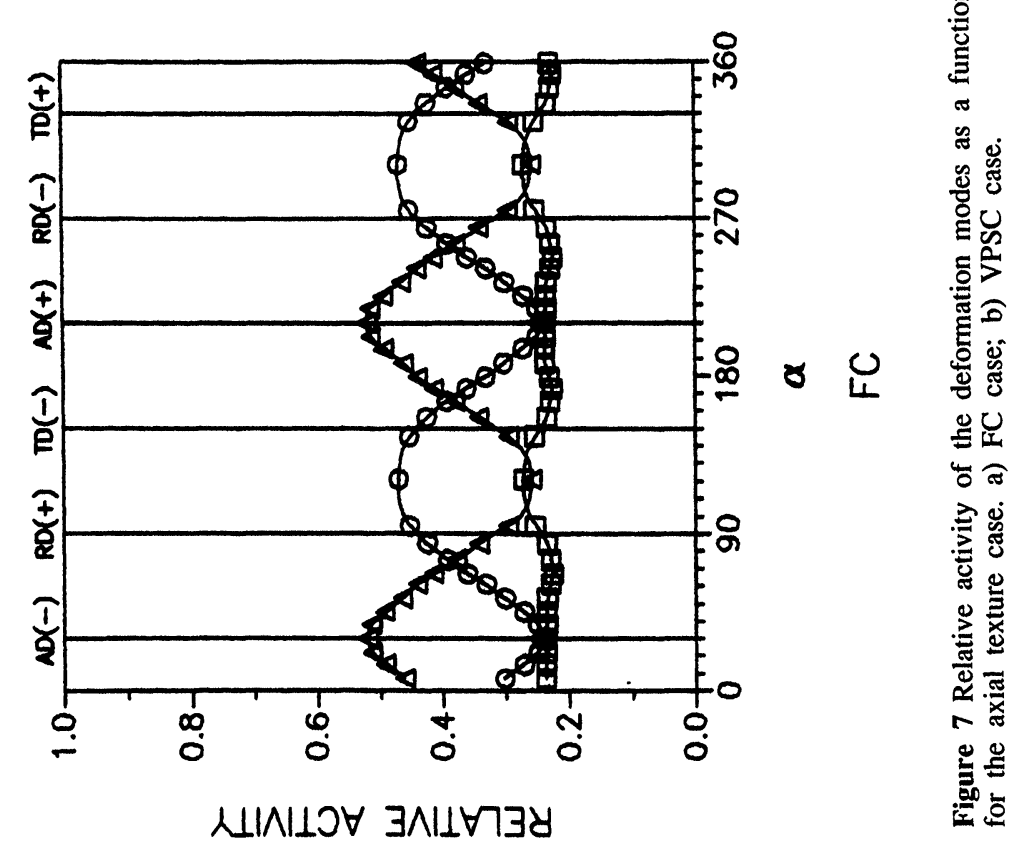


Table 7 Set of irreducible vertices associated with the SCYS of an hcp crystal for $\tau^{\text {preas }}=1.0$, $\tau^{b<2}=1.5, \tau^{p y r<c+a>}=4.0$.

\begin{tabular}{|c|c|c|c|c|c|c|c|c|c|c|c|c|c|c|c|c|c|c|c|}
\hline \multirow[t]{2}{*}{ \# } & \multicolumn{8}{|c|}{$+O T+O P+P O+P Q+Q \begin{array}{c}p y r<c+a> \\
\\
+Q Q R+R Q\end{array}$} & & $S+S R$ & \multicolumn{3}{|c|}{$+S T+T S+T O$} & \multicolumn{3}{|c|}{$\begin{array}{r}p r<a> \\
+E B+F B+G B\end{array}$} & \multicolumn{3}{|c|}{$\begin{array}{r}b<a> \\
+B E+B F+B G\end{array}$} \\
\hline & 1 & 1 & 1 & 1 & & & 1 & 1 & 1 & 1 & 1 & 1 & 1 & 0 & 0 & 0 & 0 & 0 & 0 \\
\hline 2 & 0 & 0 & 0 & 0 & & & 0 & 0 & 0 & 0 & 1 & 1 & 1 & 0 & $\overline{1}$ & 0 & 0 & 0 & 0 \\
\hline 3 & 0 & 0 & 0 & 0 & & & 1 & 0 & 0 & 0 & 0 & 1 & 1 & 1 & $\overline{1}$ & 0 & 0 & 0 & 0 \\
\hline 4 & 0 & 0 & 0 & 1 & & & 0 & 0 & 0 & 0 & 1 & 1 & 0 & 0 & $\overline{1}$ & 1 & 0 & 0 & 0 \\
\hline 5 & 0 & 0 & 0 & 0 & & & 0 & 1 & 0 & 1 & 1 & 0 & 1 & 0 & 0 & 0 & $\overline{1}$ & 0 & 1 \\
\hline 6 & 0 & 0 & 0 & 0 & & & 0 & 1 & 1 & 1 & 1 & 0 & 0 & 0 & 0 & 0 & 0 & 0 & 1 \\
\hline 7 & 0 & 0 & 0 & 0 & & & 0 & 0 & 0 & 1 & 1 & 0 & 0 & $\overline{1}$ & 0 & 1 & $\overline{1}$ & 0 & 1 \\
\hline 8 & 0 & 0 & 0 & 0 & & & 0 & 1 & 0 & 0 & 1 & 0 & 1 & 1 & $\underline{1}$ & 0 & 0 & 0 & 0 \\
\hline 9 & 0 & 0 & 0 & 0 & & & 0 & 0 & 0 & 0 & 1 & 0 & 1 & 1 & $\frac{1}{1}$ & 0 & 0 & 0 & 1 \\
\hline 10 & 0 & 0 & 0 & 0 & & & 0 & 1 & 0 & 0 & 0 & 0 & 1 & $\underline{1}$ & 0 & $\overline{1}$ & $\overline{1}$ & 0 & 1 \\
\hline 11 & 0 & 0 & 0 & 0 & & & 0 & 0 & 1 & 1 & 0 & 0 & 0 & $\frac{2}{1}$ & 1 & 0 & 0 & 0 & 1 \\
\hline 12 & 0 & 0 & 0 & $\overline{1}$ & & & 0 & 0 & 0 & 0 & 0 & 0 & 0 & 0 & 1 & $\overline{1}$ & $\overline{1}$ & 0 & 1 \\
\hline 13 & 0 & 0 & 0 & 0 & & & 0 & 0 & 0 & 0 & 0 & 0 & 1 & 1 & $\frac{2}{1}$ & 0 & $\overline{1}$ & 0 & 1 \\
\hline 14 & 0 & 0 & 0 & 0 & & & 0 & 1 & 1 & 1 & 0 & 0 & 0 & 0 & 1 & 0 & 0 & 0 & 1 \\
\hline 15 & 0 & 0 & 0 & 0 & & & 0 & 1 & 0 & 0 & 1 & 0 & 1 & 1 & 0 & 0 & 0 & 0 & 1 \\
\hline 16 & 0 & 0 & 0 & 0 & & & 0 & 0 & 0 & 0 & 1 & 0 & 1 & 0 & $\overline{1}$ & 0 & $\overline{1}$ & 0 & 1 \\
\hline 17 & 0 & 0 & 0 & 0 & & & 0 & 1 & 0 & 0 & 1 & 0 & 0 & 1 & $\hat{\overline{1}}$ & 0 & 0 & 0 & 1 \\
\hline
\end{tabular}

\section{CONCLUSIONS}

The severe plastic anisotropy of hcp crystals at low temperature is a challenging feature for testing the performance of polycrystal models. Here we use a micro-macro approach to polycrystal plasticity to demonstrate the role of twinning as the mechanism responsible for a texture-induced strength differential observed in heavily textured hcp polycrystals. This result is a consequence of the plastic properties of the aggregate alone, since the formulation does not account for elastic or internal stress effects (such as Bauschinger effect). We show that the FC assumption leads to unrealistic results since it tends to suppress the manifestation of the highly directional properties of the hexagonal crystals at the polycrystal level. On the other hand, we show that using a VPSC model and typical low-temperature deformation modes (i.e.: including twinning modes), we are able to reproduce the strength differential. In addition, within the VPSC model deformation is mostly accommodated by the systems with lower CRSS, and the harder pyramidal slip or twinning systems make a significant contribution only when the applied stress is aligned with the basal poles texture component.

We believe that these results are relevant is connection with recent efforts devoted to the development of "hybrid" numerical codes. The later simulate forming operations using finite element methods coupled with polycrystalline models to calculate the constitutive response (see, for instance, Dawson et al., 1994). Our calculations show that the effect of texture on the plastic anisotropy of hcp materials can be well reproduced by means of a VPSC approach. 
Besides the viscoplastic approach, a model based on an elastoplastic formulation could also be applied to the calculation of the yield behavior of a textured polycrystal. An elastoplastic model for PCYS calculation requires to define a precise value for the offset strain associated with the initiation of macroplasticity. As shown by Canova (1988), the shapes of the PCYS calculated by means of an elastoplastic model can differ drastically depending on the offset strain criterion chosen. The elastoplastic and viscoplastic results become comparable when the offset strain is selected to be large enough for assuring a fully plastic condition in all the grains. A comparison between elastoplastic and viscoplastic approaches for PCYS calculation together with the role of the internal stresses on the yield behavior of hcp polycrystals are subjects of a future paper (Turner et al., 1996).

\section{References}

Akhtar A. (1973). Basal Slip in Zirconium. Acta Met., 21, 1.

Canova G. R. and Kocks U. F. (1984). The Development of Deformation Textures and Resulting Properties of fcc Metals. In Proc. 7th. Int. Conf. on Textures of Materials (ICOTOM-7), edited by C. M. Brakman, P. Jongenburger and E. J. Mittemeijer, pp. 651-658. Zwijndrecht: Netherlands Society for Materials Science.

Canova G. R., Kocks U. F., Tomé C. N. and Jonas J. J. (1985). The Yield Surface of Textured Polycyrstals. J. Mech. Phys. Solids, 33, 371.

Canova G. R. (1988). Effect of Texture and Grain Shape on Anisotropy. Revue Phys. Appl., 23, 533.

Dawson P. R., Beaudoin A. J., Mathur K. K. (1994). Finite Element Modeling of Polycrystalline Solids. Materials Science Forum, 157-162, 1703.

Honneff H. and Mecking H. (1981). Analysis of Deformation Texture at Different Rolling Conditions. In Proc. 6th. Int. Conf. on Textures of Materials (ICOTOM-6), edited by S. Nagashima, pp. 347-355. Tokio: The Iron and Steel Inst. of Japan.

Hosford W. F. (1993). The mechanics of Crystals and Textured Polycrystals. New York: Oxford Science Publications.

Kelly E. W. and Hosford W. F. (1968). Deformation Characteristics of Textured Magnesium. Trans. TMS-AIME, 242, 654.

Lebensohn R. A. and Tomé C. N. (1993). A selfconsistent Anisotropic Approach for the Simulation of Plastic Deformation and Texture Development of Polycrystals: Application to Zirconium Alloys. Acta Metall. Mater. 41, 2611.

Lebensohn R. A., Sanchez P. V. and Pochettino A. A (1994). Modelling Texture Development Zirconium Alloys at High Temperatures. Scripta Metall. Mater. 30, 481.

Lebensohn R. A. and Tome C. N. (1994). A selfconsistent Viscoplastic Model: Prediction of Rolling Textures of Anisotropic Polycrystals. Mat. Sc. Eng. A, A175, 71.

Lee D. and Backofen W. A. (1966). An Experimental Determination of the Yield Locus for Titanium and Titanium Alloys Sheet. Trans. TMS-AIME, 236, 1077.

Molinari A., Canova G. R. and Ahzi S. (1985). A Selfconsistent Approach of the Large Deformation Polycrystal Viscoplasticity. Acta Metall., 35, 2983.

Picklesimer M. L. (1966). Deformation, Creep and Fracture in Alpha-Zirconium Alloys. Electrochemical Technology, 4, 289.

Pochettino A. A., Gannio N., Vial-Edwards C. and Penelle R. (1992). Texture and Pyramidal Slip in $\mathrm{Ti}, \mathrm{Zr}$ and Their Alloys. Scripta Metall. Mater., 27, 1859.

Tenckhoff E. (1974). Operable Deformation Systems and mechanical Behavior of Textured Zircaloy Tubing. In Zirconium in Nuclear Applications, ASTM STP, 551, 179.

Tenckhoff E. (1978). The Development of the Deformation Texture in Zirconium During Rolling in Sequential Passes. Met. Trans. A, 9A, 1401.

Tomé C. N. and Kocks U. F. (1985). The Yield Surface of hcp Crystals. Acta Metall., 33, 603.

Tomé C. N., Pochettino A. A. and Penelle R. (1988). Analysis of Plastic Anisotropy of Rolled Zircaloy4. In Proc. 8th. Int. Conf. on Textures of Materials (ICOTOM-8), edited by J.S. Kallend and G. Gottstein, pp. 985-990. Warrendale: The Metallurgical Society.

Tomé C. N., Lebensohn R. A. and Kocks U. F. (1991). A Model for Texture Development Dominated by Deformation Twinning: Application of Zirconium Alloys. Acta Metall. Mater., 39, 2667.

Turner P. A., Signorelli J. W., Lebensohn R. A. and Tomé C. N. (1996), to be published. 